\title{
Effects of random food deprivation and refeeding on energy metabolism, behavior and hypothalamic neuropeptide expression in Apodemus chevrieri
}

Zhu Wan-long* Wang Zheng-kun

\begin{abstract}
Maintaining adaptive control of behavior and physiology is the main strategy used by animals in responding to changes of food resources. To investigate the effects of random food deprivation (FD) and refeeding on energy metabolism and behavior in Apodemus chevrieri, we acclimated adult males to FD for 4 weeks, then refed them ad libitum for 4 weeks (FD-Re group). During the period of FD, animals were fed ad libitum for 4 randomly assigned days each week, and deprived of food the other 3 days. A control group was fed ad libitum for 8 weeks. At 4 and 8 weeks we measured body mass, thermogenesis, serum leptin levels, body composition, gastrointestinal tract morphology, behavior and hypothalamic neuropeptide expression. At 4 weeks, food intake, gastrointestinal mass, neuropeptide Y (NPY) and agouti-related protein (AgRP) mRNA expressions increased and thermogenesis, leptin levels, pro-opiomelanocortin (POMC) and cocaine- and amphetamine-regulated transcript (CART) expressions decreased in FD compared with controls. FD also showed more resting behavior and less activity than the controls on ad libitum day. There were no differences between FD-Re and controls at 8 weeks, indicating significant plasticity. These results suggested that animals can compensate for unpredictable
\end{abstract}


1 reduction in food availability by increasing food intake and reducing energy expended through

2 thermogenesis and activity. Leptin levels, NPY, AgRP, POMC, and CART mRNA levels may

3 also regulate energy metabolism. Significant plasticity in energy metabolism and behavior was

4 shown by $A$. chevrieri over a short timescale, allowing them to adapt to food shortages in

$5 \quad$ nutritionally unpredictable environments.

6 Key words: Apodemus chevrieri; behavioral patterns; hypothalamic neuropeptide; serum leptin 7 levels

\section{Introduction}

Physiological adaptability, including adjustments of body mass and energy metabolism, is one of the main strategies used by animals to respond to fluctuations in their natural environment, significantly enhancing their ability to survive (Zhao and Wang, 2007). Availability of food resources affect animal survival, reproduction and evolution (Bacigalupe and Bozinovic, 2002). Faced with food restrictions, deprivation or hunger, small rodents may reduce body mass, body temperature, energy expenditure, thermogenesis and/or begin to store food (Zhan et al., 2009). Many studies suggested that small mammals adapt to food shortage through adjustments in body mass and energy balance, and food restriction has obvious effects on physiological characteristics, including reductions in energy consumption and changes to their digestive tracts (Bozinovic et al., 2007). Food restriction reduced body mass, body fat mass, metabolic rate and serum leptin levels in Lasiopodomys brandtii (Zhan et al., 2009) and random food deprivation reduced body mass, thermogenesis in Eothenomys miletus (Zhu et al., 2014). However, while body mass in rats and mice increased significantly after re-feeding (Friedman and Halaas, 1998; Woods et al., 1998), but Mesocricetus auratus (Schneider et al., 2000) and Phodopus sungorus (Day and Bartness, 2003) 
1 did not increased body mass after refeeding. The phenotype of digestive tracts can reflect

2 physiological and ecological adaptations made by animals to their environment (Pucek, 1965).

3 Food restrictions have been shown to induce changes in the morphology of the digestive tract

4 (Bozinovic, 2007).

Resting metabolic rate (RMR) and nonshivering thrmogenesis (NST) are two important

modes of energy expenditure in small mammals, and are greatly influenced by environmental

7 factors (Zhao and Wang, 2007). Changes in food quantity and/or quality lead to variations in

8 metabolic energy expenditure (Veloso and Bozinovic, 1993), and many studies have demonstrated

9 that the low quality food reduced RMR in rodents (Hambly and Speakman, 2005; Zhao and Wang,

2007). Many animals also actively or passively regulate behavior to adapt to changes in

11 environmental conditions. In response to food restriction, Mus musculus (Blank and Desjardins, 1985) and rats (Sherwin, 1998) increased their activity levels, while MF1 mice (Hambly and Speakman, 2005) and Eutamias minimus (Cornish and Mrosovsky, 1965) reduced activity levels, suggesting that behavioral responses to food restriction can be species-specific.

Leptin, a $16-\mathrm{kDa}$ protein, contributes to the maintenance of body mass by influencing food intake and energy expenditure (Friedman and Hallas 1998). A positive correlation has been observed between serum leptin levels and body fat mass in many small mammals (Abelenda et al. 2003). The hypothalamic arcuate nucleus (ARC) can regulate food intake in response to environmental changes (Heijboer et al., 2005; Aguilar et al., 2011). Within the ARC are two types of neuropeptides: orexigenic neuropeptides including neuropeptide Y (NPY) and agouti-related protein (AgRP); and anorectic neuropeptides including pro-opiomelanocortin (POMC) and cocaine- and amphetamine-regulated tran-script (CART). The balance between orexigenic and 
anorectic neuron activities can influence food intake and energy expenditure in mammals

2 (Friedman and Halaas, 1998). Leptin is mediated by a hierarchy of orexigenic and anorectic

3 neuropeptidergic neurons located in specific sites in the hypothalamus (Arch, 2005). In a state of

4 negative energy balance, a reduction in leptin stimulates NPY and AgRP expressions and suppresses POMC and CART expressions, eliciting an increase in food intake and a reduction in energy expenditure (Xiao et al., 2005). Studies showed that when rats are fed with a high fat food,

7 NPY expression and body mass are increased (Toshihiro and Akio, 1993). Prolonged hunger also 8 increases NPY expression in rats (Sucajtys-Szulc et al., 2008); and food-restricted lactating rats have higher NPY levels than those fed ad libitum (Abizaid et al., 1997). Prolonged food restriction reduces body mass, body fat mass, POMC and CART expressions, and increases AgRP

11 expressions in sheep (Henry et al., 2001). Phodopus sungorus showed increased AgRP expression during a food shortage (Mercer et al., 2000). Fasting can also reduce CART expression (Tian et al., 2004), for instance mice showed reduced CART expression and increased appetite following a 48-hour fast (Yoo et al., 2011).

Chevrier's field mouse, Apodemus chevrieri (Mammalia: Rodentia: Muridae) is endemic to the Hengduan mountains region of China (Zheng 1993). A. chevrieri has been reported to show seasonal changes in body mass and digestive tract morphology (Zhu et al., 2012). During cold exposure, A. chevrieri showed increased energy intake, thermogenesis and reduced body mass, and serum leptin levels (Zhu et al., 2011). Food restriction alone may also reduce body mass in $A$. chevrieri (Zhu et al., 2013). However, the effects of random food deprivation (FD) on serum leptin levels, behavior, hypothalamic neuropeptide expression, and their influence on body mass regulation and thermogenesis in $A$. chevrieri remain unknown. In the present study, we examined 
the effects of FD and refeeding on energy metabolism and behavior in Apodemus chevrieri adult males, we measured body mass, thermogenesis, serum leptin levels, body composition, gastrointestinal tract mass and length, behavior and hypothalamic neuropeptide expression after 4 weeks of FD and again after 4 weeks of refeeding. We hypothesized that $A$. chevrieri would respond to FD by reducing body mass, body fat mass, and thermogenesis in association with reduced serum leptin levels, but energy metabolism would return to normal after refeeding, demonstrating phenotypic plasticity. We predicted that leptin levels, NPY, AgRP, POMC, and CART mRNA levels would play a role in the regulation of energy metabolism in A. chevrieri.

\section{Materials and Methods}

\subsection{Animals and sampling}

A. chevrieri were obtained from a laboratory colony, founded by animals captured from farmland $\left(26^{\circ} 15^{\prime}-26^{\circ} 45^{\prime} \mathrm{N}\right.$; $99^{\circ} 40^{\prime}-99^{\circ} 55^{\prime} \mathrm{E}$; altitude 2,590m) in Jianchuan County, Yunnan province. Adult male A. chevrieri (120 days of age) were housed individually in plastic boxes $\left(26 \times 16 \times 15 \mathrm{~cm}^{3}\right)$. Animals were kept in a room temperature of $25 \pm 1{ }^{\circ} \mathrm{C}$ with a photoperiod of 12L:12D (with lights on at 08:00 h), and provided food (standard rabbit chow produced by Kunming Medical University, Kunming) and water ad libitum. After 1 month of stabilization, animals were randomly assigned to a control group or a random food deprivation refeeding group (FD-Re). Controls $(\mathrm{n}=20)$, were fed ad libitum during 8 weeks, while FD-Re $(\mathrm{n}=20)$ were deprived of food for a random 3 days each week and fed ad libitum on the other 4 days for a total of 4 weeks, then fed ad libitum for a further 4 weeks (Fig 1) (Barboza et al., 2001; 2002). After 4

21 weeks, 10 animals were randomly selected from each group (4W Control group and 4W FD group) 
1 for the measurement of RMR, NST, serum leptin levels, body composition, gastrointestinal tract

2 mass and length, behavior and hypothalamic neuropeptide expression. These measurements were

3 taken again from the remaining 10 animals of each group (8W Control group and $8 \mathrm{~W}$ FD-Re

4 group) at 8 weeks. All animals were sacrificed between $0900 \mathrm{~h}$ and $1100 \mathrm{~h}$ by decapitation, and

5 animals were dissected to evaluate organ morphology. Blood was centrifuged at 4,000 rpm for 30

6 min after a 30 min interval. Blood serum was collected and stored at $-75^{\circ} \mathrm{C}$ prior to hormone

7 measurement. During the 8 weeks of experiment, food intake was measured daily and body mass

8 was measured in 3 days. All animals were dissected to evaluate organ morphology, and young

9 individuals were excluded in present study. All animal procedures were approved by the Animal

10 Care and Use Committee of Life of Science, Yunnan Normal University (13-0901-011).

112.2 Morphology

\subsection{Food intake}

Immediately after sacrifice at 4 and 8 weeks, trunk blood was collected and visceral organs including liver, brown adipose tissue (BAT), heart, lung, kidneys, spleen and gastrointestinal tract (stomach, small intestine, caecum, large intestine) were excised and weighed $( \pm 1 \mathrm{mg})$. The stomach and intestines were rinsed with saline to remove gut contents and then weighed. The remaining carcass and the excised organs were dried to constant mass in an oven at $60{ }^{\circ} \mathrm{C}$ (for at least $72 \mathrm{~h}$ ), and then weighed again to obtain a dry mass. Total body fat mass was extracted from the dried carcass by ether extraction in a Soxhlet apparatus (Zhang and Wang 2007). 
metabolic cage $\left(20 \times 15 \times 15 \mathrm{~cm}^{3}\right)$ with no nest materials, and fed standard rabbit chow. Animals were fed at a set time (at $1100 \mathrm{~h}$ ), and residual food collected was collected the following day. Residual food was dried in a vacuum dryer to a constant weight, which was recorded.

\subsection{Measurement of metabolic rates}

Metabolic rates were measured using an AD ML870 open respirometer (AD Instruments, Australia) at $25{ }^{\circ} \mathrm{C}$ within the thermal neutral zone, and gas analysis was performed using a ML206 gas analysis instrument (AD Instruments). The temperature was controlled using a SPX-300 artificial climatic incubator $\left( \pm 0.5^{\circ} \mathrm{C}\right)$ (Changsha, China), the metabolic chamber volume was $500 \mathrm{ml}$ and airflow rate was $200 \mathrm{ml} / \mathrm{min}$. Animals were stabilized in the metabolic chamber for at least 60 min prior to the RMR measurement, and oxygen consumption was recorded for at least $120 \mathrm{~min}$ at $1 \mathrm{~min}$ intervals. Ten stable consecutive low readings were taken to calculate RMR following Li and Wang (2005), using the method for calculating the metabolic rate provides by Hills (1972).

\footnotetext{
Nonshivering thermogenesis (NST) was induced by a subcutaneous injection of norepinephrine (NE) (Shanghai Harvest Pharmaceutical Co. Ltd, China) and measured at $25{ }^{\circ} \mathrm{C}$. Two consecutive high oxygen consumption readings from each 60 -min measurement were taken to calculate NST (Li and Wang, 2005). The doses of norepinephrine were approximately $0.8-1.0$ $\mathrm{mg} / \mathrm{kg}$, according to dose-dependent response curves generated before the experiment and using the equation of Heldmaier (1971).
}

\subsection{Behavioral observations}

Behaviors were observed on day 27 (FD food deprivation day), day 28 (FD feeding day) and 
day 56. The dominant behavior was classified into one of four categories: general activity, feeding behavior, grooming behavior and resting behavior (Speakman et al. 2001). General activity denoted any active movements such as walking around the cage and climbing on the cage bars, feeding behavior denoted eating but not drinking; grooming denoted self-grooming and resting denoted inactivity in any area of the cage. Animals were observed in sequence for $30 \mathrm{~s}$ each over the course of an 8-min session, during which the dominant behavior was recorded as one of the four aforementioned types. Observation was made during period of $0800-1200 \mathrm{~h}$ and $1800-2200$ h, respectively. Each animal was observed 60 times in $8 \mathrm{~h}$ and the percentage time spent on each behavior was calculated for each animal (Speakman et al. 2001). Observations during the dark phase were made using a $30 \mathrm{~W}$ red light (Zhao and Cao 2009).

\subsection{Measurement of serum leptin levels}

Serum leptin levels were determined by radioimmunoassay (RIA) with the ${ }^{125}$ I Multi-species Kit (Cat. No. XL-85K, Linco Research Inc.). The lowest level of leptin that can be detected by this assay was $1.0 \mathrm{ng} / \mathrm{ml}$ when using a $100-\mu l$ sample, and the inter- and intra-assay variability for leptin RIA were $<3.6 \%$ and $8.7 \%$, respectively.

\subsection{Measurements of hypothalamic neuropeptide gene expression}

Total RNA was isolated from the hypothalamus using TRIzol Kit (Invitrogen, Carlsbad, CA, USA) according to the manufacturer's instructions. To remove any contaminating DNA, RNA samples were treated with DNase I (Promega, USA) at $37{ }^{\circ} \mathrm{C}$ for $30 \mathrm{~min}$ followed by another cycle of TRIzol extraction to eliminate residual DNase I. An amount (3 $\mu \mathrm{g})$ of total RNA was transcribed into first strand cDNA for each sample using the M-MLV First Strand Kit (Invitrogen) according to the manufacturer's instructions. 
2 A. chevrieri. Species-specific primers were designed in real-time q-PCR for $\beta$-actin and the four

3 hypothalamic genes are shown in Table 1 and verified effectively in A. chevrieri (Zhang et al.,

4 2015). Standard curves were constructed for each gene using serial dilutions of cDNA (1 to

526 -fold dilutions). A comparison of the standard curves of the target genes and $\beta$-actin revealed

6 that they had similar amplification efficiencies, validating the comparative quantity method used.

7 Real-time q-PCR was performed using a SYBR Green I qPCR kit (Invitrogen) and the ABI

8 Prism $^{\circledR} 7000$ Sequence Detection system (Applied Biosystems, Carlsbad CA, USA). Real-time

9 qPCR was carried out in $20-\mu \mathrm{L}$ reactions comprising $9.5 \mu \mathrm{L}$ RNase-free $\mathrm{ddH}_{2} \mathrm{O}, 9.0 \mu \mathrm{L}$ Platinum ${ }^{\circledR}$

10 Quantitative PCR SuperMix-UDG (including Rox), $0.5 \mu \mathrm{L}$ cDNA templates, $0.5 \mu \mathrm{L} 10 \mu \mathrm{moL} / \mathrm{L}$

11 forward primer, and $0.5 \mu \mathrm{L} 10 \mu \mathrm{moL} / \mathrm{L}$ reserse primer. Each sample was analyzed in triplicate.

12 Thermal cycling conditions were: $50^{\circ} \mathrm{C}$ for $120 \mathrm{~s}, 95{ }^{\circ} \mathrm{C}$ for $120 \mathrm{~s}, 45$ cycles of $95{ }^{\circ} \mathrm{C}$ for $15 \mathrm{~s}$, 13 and $60{ }^{\circ} \mathrm{C}$ for $45 \mathrm{~s}$.

14

Table 1 Gene-Specific Primers Used for Real-Time qPCR.

\begin{tabular}{lll}
\hline Primer & Oligonuncleotide sequence (5' to 3') & Product size (bp) \\
\hline NPY (forward) & TCGCTCTGTCCCTGCTCGTGTG & 130 \\
NPY(reverse) & TCTCTTGCCGTATCTCTGCCTGGTG & \\
AgRP(forward) & AGAGTTCTCAGGTCTAAGTCT & 190 \\
AgRP(reverse) & CTTGAAGAAGCGGCAGTAGCACGT & \\
POMC(forward) & CCTGTGAAGGTGTACCCAATGTC & 241 \\
POMC(reverse) & CACGTTCTTGATGATGGCGTTC & \\
CART(forward) & AGAAGAAGTACGGCCAAGTCC & 50 \\
CART(reverse) & CACACAGCTTCCCGATCC & \\
$\beta$-actin(forward) & TCGCTCTGTCCCTGCTCGTGTG & 170 \\
$\beta$-actin (reverse) & TCTCTTGCCGTATCTCTGCCTGGTG & \\
\hline
\end{tabular}


Data were analyzed using the software package SPSS 15.0. Prior to all statistical analyses,

3 data were examined for normality and homogeneity of variance using Kolmogorov-Smirnov and

4 Levene tests, respectively. Body mass and food intake were analyzed using repeated measures

5 ANOVA. RMR, NST, body composition and hypothalamic neuropeptide gene expression were analyzed using One-way ANCOVA, with body mass or carcass mass as a covariate, followed by

7 Tukey's HSD post-hoc tests. Categorized behavioral data were analyzed using

8 independent-samples t-test, with an arcsine-square-root transformation performed on the

9 percentage data prior to analysis. To detect any associations of serum leptin levels with body fat mass, we used Pearson-correlation analysis. Results are presented as means \pm SEM and $\mathrm{P}<0.05$

11 was considered to be statistically significant.

\section{Result}

\subsection{Body mass}

There were no differences in body mass between control and FD-Re groups on day $0(t=0.12$, $\mathrm{P}>0.05$ ). On day 28 , body mass was significantly reduced in the FD-Re group compared with that of controls (FD-Re: $28.32 \pm 0.32 \mathrm{~g}$, control: $33.72 \pm 0.41 \mathrm{~g}, \mathrm{t}=4.25, \mathrm{P}<0.01$ ). On day 56 , body mass in $8 \mathrm{~W}$ FD-Re group had returned to control levels, with no significant differences between two groups ( $\mathrm{t}=0.024, \mathrm{P}>0.05$, Fig 2$)$.

\subsection{Body composition and gastrointestinal tract mass and length}

FD had a significant effect on carcass mass, body fat mass, wet liver mass and wet BAT mass by day 28 (Table 2), but had no effect on our other measures of body composition ( $\mathrm{P}>0.05)$. FD also had a significant effect by day 28 on the mass of the gastrointestinal tract with gut content and 
1 the length of small intestine, but did not affect other gastrointestinal tract measurements $(\mathrm{P}>0.05$,

2 Table 3).

$3 \quad 3.3$ Food intake

There were no differences of food intake between control and FD-Re groups on day $0(\mathrm{t}=0.25$, $\mathrm{P}>0.05$ ). During the 4 weeks of FD, food intake in $4 \mathrm{~W}$ FD group increased significantly $(\mathrm{t}=-5.65$, $\mathrm{P}<0.01$ ). During the subsequent 4 weeks of refeeding, FD-Re food intake decreased, returning to the control levels on day $34(\mathrm{t}=0.19, \mathrm{P}>0.05)$. Food intake showed significantly changes with time in FD-Re group ( $\mathrm{F}=26.32, \mathrm{P}<0.01$, Fig 3), and no differences with time in the control group $(\mathrm{F}=0.68, \mathrm{P}>0.05$, Fig 3$)$.

\subsection{RMR and NST}

On day 28 , RMR was $13.93 \%$ lower in the FD-Re group than that of the control group (FD-Re: $2.41 \pm 0.11 \mathrm{mlO}_{2} /$ g.h, control: $2.80 \pm 0.14 \mathrm{mlO}_{2} /$ g.h), but returned to control levels by day $56(\mathrm{~F}=2.43, \mathrm{P}<0.05$, Fig 4). NST was also reduced in the FD-Re group at day 28 , but returned to control levels by day $56(\mathrm{~F}=2.56, \mathrm{P}<0.05$, Fig 5$)$.

\subsection{Behavior}

On day 27 (a food deprivation day), the percentage of time spent on general activity and resting was greater in the FD-Re group than that of the control group (activity: $\mathrm{t}=3.21, \mathrm{P}<0.01$; resting: $\mathrm{t}=1.74, \mathrm{P}<0.05$ ), and that spent on grooming was lower in FD-Re group than that in control group ( $\mathrm{t}=-2.15, \mathrm{P}<0.05$, Fig $6 \mathrm{~A}$ ). On day 28 (feeding day), the percentage of time spent feeding and resting behavior were higher in FD-Re group than that in control group (feeding: $\mathrm{t}=2.27, \mathrm{P}<0.05$; resting: $\mathrm{t}=3.02, \mathrm{P}<0.01$ ), and that spent activity and grooming was lower in FD-Re group than that in control group (activity: $\mathrm{t}=-4.35, \mathrm{P}<0.01$; grooming: $\mathrm{t}=-6.98, \mathrm{P}<0.01$, Fig $6 \mathrm{~B}$ ). 
1 On day 56, there were no differences in the percentage time spent on different behaviors between

2 the two groups $(\mathrm{P}>0.05$, Fig 6C).

33.6 Serum leptin level and hypothalamic neuropeptide gene expression

Serum leptin levels was reduced in the FD-Re group at day 28 compared with controls (FD-Re: $0.98 \pm 0.03 \mathrm{ml} \mathrm{O}_{2} /$ g.h, controls: $1.51 \pm 0.06 \mathrm{ml} \mathrm{O}_{2} /$ g.h), but returned to control levels by day $56(\mathrm{~F}=2.13, \mathrm{P}<0.05$, Fig 7$)$. There was a positive correlation between body fat mass and serum leptin levels $(r=0.625, \mathrm{P}<0.01)$. FD had significant effects on NPY, AgRP, POMC and CART expressions (NPY: F=2.25, $\mathrm{P}<0.05 ;$ AgRP: $\mathrm{F}=3.36, \mathrm{P}<0.01 ; \mathrm{POMC}: \mathrm{F}=3.19, \mathrm{P}<0.01$; CART: $\mathrm{F}=2.85, \mathrm{P}<0.01$, Fig 8). NPY and AgRP expressions were increased and POMC and CART expressions were reduced on day 28 in FD-Re group compared with controls, but returned to control levels on day 56.

\section{Discussion}

4.1Body mass and body composition

Body mass is an important index of animal nutritional status, and its stability relies on energy balance (Zhao and Wang, 2007). In the present study, FD reduced body mass significantly in $A$. chevrieri, which returned to control levels after refeeding, as shown by other rodent models (Trayhurn and Jennings, 1988; Kouda et al., 2004). The reduction in body mass was associated with reduced carcass mass and body fat mass (Mustonen et al., 2005). With the exception of wet liver mass and BAT mass, organ mass was not affected by FD, suggesting that $A$. chevrieri maintain energy expenditure by reducing carcass mass, liver and BAT mass use. After refeeding, carcass mass and body fat mass returned to control levels, which probably contributed to the recovery of body mass (Freminet, 1981). 
Small mammals, such as Meriones shawi, usually increase food intake under conditions of

3 food restriction, (Demas and Bartness, 1999). When faced with large environmental fluctuations in

4 food quality and/or quantity, increasing food intake can improve their survival ability and reduce their risk of predation, though some animals do not use this strategy. In our study, food intake increased significantly in the FD-Re group compared with the control group during the period of FD, but returned to the control levels after refeeding. The digestive tract also showed significant changes with FD, with greater small intestine length and greater digestive tract mass with gut content in the FD group than that of control group. These results indicated that $A$. chevrieri can make full use of occasional feeding opportunity by increasing food intake to compensate for

11 reduced food availability. Similar results have also been reported for other animals (Naya et al. 2005; 2008; Zhao and Wang 2007). The return of food intake to control levels after refeeding

13 suggests that digestive tract physiological plasticity plays an important roles in body mass regulation in animals (Zhao et al., 2009).

RMR and NST consume significant amounts of energy in small mammals, which can reflect overall levels of energy consumption in different species or individuals. RMR and NST are affected by temperature, food, the activity levels as well as other factors (Terblanche et al., 2007). In the present study, FD significantly reduced RMR and NST. This suggests that food deprived $A$. chevrieri could not consume enough energy to meet their requirements, so RMR and NST were lowered to reduce energy consumption to maintain the body's normal physiological activities. Hunger has previously been shown to reduce the metabolic rate in rats and mice (DeLaney et al., 
1 1999). Liver is the major organ for RMR, and BAT is the major organs for NST

2 (Himms-Hagen,1990). In the present study, the reduction of RMR and NST in FD group may be

3 affected by reduced liver and BAT mass. RMR and NST returned to the control levels after

4 refeeding, as did liver and BAT mass, demonstrating remarkable plasticity.

\subsection{Behavior}

Animals often change their behavior to adapt to changes in environmental conditions (Zhao and Cao 2009). In the present study, the level of general activity was significantly higher in FD group than that of controls on a food deprivation day, as also shown in mice (Sherwin 1998), indicating that animal increase activity to look for food when the food resources are limited (Alvarenga et al. 2005). On ad libitum day, FD group animals spent a greater percentage of time feeding than controls, suggesting that animal can reduce general activity, increased feeding behavior and reduce energy expenditure to compensate for a shortage of food (Gursoy et al. 2001). Moreover, our results indicated that on an ad libitum feeding day, FD-Re animals reduced their energy expenditure by reducing general activity and increasing resting behavior, and increased their feeding behavior to increase food intake. After refeeding, the percentage time spent on activity, grooming, feeding and rest behavior all returned to the control levels in the FD-Re group, showing significant plasticity and suggesting that this plasticity of behavior plays an important role in the adaptation of small mammals to food shortages.

\subsection{Serum leptin level and hypothalamic neuropeptide gene expression}

Leptin plays an important role in body mass regulation (Friedman and Hallas 1998), which suggests that leptin levels can reflect content of body fat mass (Schneider et al., 2000). We found a positive correlation between serum leptin levels and body fat mass in the present study, supporting 
1 the claim that leptin can act as a lipid signaling molecule (Zhao and Wang, 2006). Orexigenic

2 neuropeptides and anorectic neuropeptides can regulate food intake in small mammals (Arch,

3 2005). In the present study, FD had significant effects on hypothalamus neuropeptide genes

4 expressions in A. chevrieri. NPY and AgRP expressions were increased to increase appetite and

5 reduce heat production. POMC and CART expressions were reduced, which also increased

6 appetite (Balaskó et al., 2006; Larsen and Hunter, 2006; Kim and Harrington, 2008; Tracy et al.,

7 2008; Lukaszewski et al., 2013; Zhao et al., 2014). These results indicated that NPY/AgRP and

8 POMC/CART expressions can regulate body mass and energy metabolism in A. chevrieri under

9 conditions of $\mathrm{FD}$.

In conclusion, FD had significant effects on body mass, food intake, behavior,

11 gastrointestinal tract mass and length and hypothalamic neuropeptide gene expression in $A$.

12 chevrieri. FD group had a significantly higher percentage of feeding behavior and lower activity

13 than control group on ad libitum day. These effects were no longer apparent after 4 weeks of ad

14 libitum refeeding, indicating significant plasticity. Our results suggest that animals can

15 compensate for an unpredictable reduction in food availability by increasing food intake and

16 reducing energy expended through thermogenesis and activity. Changes in leptin levels, NPY,

17 AgRP, POMC, and CART mRNA levels may play a role in regulating energy metabolism .

18 Acknowledgments

This research was financially supported by National Science Foundation of China (No. 31260097; 31560126),

20 and Launch scientific research projects of Yunnan Normal University. We wish to thank Pro. Burkart Engesser at

21 Historisches Museum Basel, Switzerland for correcting the English usage in the draft. Thank you for the two 


\section{References}

2

Abelenda. M, Ledesma, A., Rial, E., Puerta, M. 2003. Leptin administration to cold-acclimated rats reduces both food intake and brown adipose tissue thermogenesis. Journal of Thermal Biology, 28: 525-530.

Abizaid, A., Walker, C.D., Woodside, B. 1997. Changes in neuropeptide Y immunoreactivity in the arcuate nucleus during and after food restriction in lactating rats. Brain Research, 761: 306-312.

Aguilar, A.J., Conde-Sieira, M., López-Patiño, M.A., Míguez, J.M., Soengas J.L., 2011. In vitro leptin treatment of rainbow trout hypothalamus and hindbrain affects glucosensing and gene expression of neuropeptides involved in food intake regulation. Peptides, 32: 232-240.

Alvarenga, T.A.F., Andersen, M.L., Papale, L.A., Antunes, I.B., Tufik, S. 2005. Influence of long-term food restriction on sleep pattern in male rats. Brain. Res., 1057: 49-56.

Arch, J.R. 2005. Central regulation of energy balance: inputs, outputs and leptin resistance. P. Nutr. Soc., 64: 39-46.

Bacigalupe, L.D., Bozinovic, F. 2002. Design, limitations and sustained metabolic rate: lessons from small mammals. J. Exp. Biol., 205: 2963-2970.

Balaskó, M., Pétervári, E., Koncsecskó-Gáspár, M., Székely, M. 2006. Cold-adaptation: Neuropeptide Y versus thermal signals in the development of hyperphagia. Journal of Thermal Biology, 31: 115-123

Barboza, P.S., Jorde, D. G. 2001. Intermittent feeding in a migratory omnivore: digestion and body composition of American black duck during autumn. Physiological and Biochemical Zoology, 74: 307-317.

Barboza, P. S., Jorde, D. G. 2002. Intermittent feeding during winter and spring affects body composition and reproduction of a migratory duck. Journal of Comparative Physiology, 172: 419-434.

Blank, J.L., Desjardins, C. 1985. Differential-effects of food restriction on pituitary testicular function in mice. Am. J. Physiol., 248: 181-189.

Bozinovic, F., Jose, L.P., Munoz, N.D., Ariovaldo, P., Cruz-Neto. 2007. Adjusting energy expenditures to energy 
supply: food availa- bility regulates torpor use and organ size in the hilean mouse-opossum Thylamy selegans.

Cornish, E.R., Mrosovsky, N. 1965. Activity during food deprivation and satiation of six species of rodents. Anim. Behav.,13: 242-248.

Day, D.E., Bartness, T.J. 2003. Fasting-induced increases in food hoarding are dependent on the foraging-effort level. Physiol. Behav., 78: 655-668.

DeLaney, J.P., Hansen, B.C., Bodkin, D.L., Hannah, J., Bray, G.A. 1999. Long-term caloric restriction reduces energy expenditure in aging monkeys. J. Gerontol. Biol. Sci., 54: 5-11.

Demas, G.E., Bartness, T.J. 1999. Effects of food deprivation and metabolic fuel utilization on food hoarding by jirds (Meriones shawi).Physiol. Behav., 67: 243-248.

Friedman, J.M., Halaas, J.I. 1998. Leptin and the regulation of body weight in mammals. Nature, 395: 763-770.

Freminet, A. 1981. Comparison of glycogen store in rats and guineapigs: effects of anaesthesia, fasting and re-feeding. Comp. Biochem. Physiol., 69: 655-663.

Gursoy, E., Cardoune, A., Hu, Y., Kalimi, M. 2001. Biological effects of long-term caloric restriction: adaptation with simultaneous administration of caloric stress plus repeated immobilization stress in rats. Soc. Exp. Biol. Med., 226: 97-102.

Hambly, C., Speakman, J.R. 2005. Contribution of different mechanisms to compensation for energy restriction in the mouse. Obes. Res., 13: 1548-1557.

Heldmaier, G. 1971. Nonshivering thermogenesis and body size in 567 mammals. J. Comp. Physiol., 73: 222-248.

Heijboer, A., Voshol, P., Donga, E., van Eden, C., Havekes, L., Romijn, J., Pijl, H., Corssmit, E. 2005. High fat diet induced hepatic insulin resistance is not related to changes in hypothalamic mRNA expression of NPY, AgRP, POMC and CART in mice. Peptides, 26: 2554-2558.

Henry, B.A., Rao, A., Ikenasio, B.A. 2001. Differential expression of cocaine- and amphetamine-regulated 
Himms-Hagen, J. 1990. Brown adipose tissue thermogenesis: interdisciplinary studies. Faseb. J. 4: 2890-2898.

Kim, H.J., Harrington, M.E. 2008. Neuropeptide Y-deficient mice show altered circadian response to simulated natural photoperiod. Brain Research, 1246: 96-100.

Kouda, K., Nakamura, H., Kohno, H., Ha-Kawa, S.K., Tokunaga, R., Sawada, S. 2004. Dietary restriction: effects of short-term fasting on protein uptake and cell death/proliferation in the rat liver. Mech. Ageing. Dev., 125: $375-380$

Larsen, P.J., Hunter, R.G. 2006. The role of CART in body weight homeostasis. Peptides, 27: 1981-1986.

Li, X.S., Wang, D.H. 2005. Seasonal adjustments in body mass and thermogenesis in Mongolian gerbils (Meriones unguiculatus): the roles of short photoperiod and cold. J. Comp. Physiol., 175: 593-600. high-fat diet. Peptides, 43: 146-154.

Mercer, J.G., Moar, K.M., Ross, A.W. 2000. Regulation of leptin receptor, POMC and AGRP gene expression by photoperiod and food deprivation in the hypothalamic arcuate nucleus of the male Siberian hamster

Mustonen, A.M., Pyykonen, T., Nieminen, P. 2005. Adiponectin and peptide Y in the fasting blue fox (Alopex lagopus). Comp. Biochem. Physiol., 140: 251-256. in the Andean toad Bufo spinulosus: hard-wired or flexible physiology? Comp. Biochem. Physiol., 140: 
1 Naya, D.E., Veloso, C., Bozinovic, F. 2008. Physiological flexibility in the Andean lizard Liolaemus bellii: seasonal changes in energy acquisition, storage and expenditure. J. Comp. Physiol., 178: 1007-1015.

Pucek, Z. 1965. Seasonal and age changes in the weight of internal organ of shrews. Acta Theriologica, 10: 369-438.

Rousseau, K., Actha, Z., Loudon, A.S.I. 2003. Leptin and seasonal mammals. J. Neuroendocrinol., 15: 409-414.

Schneider, J.E., Blum, R.M., Wade, G.N. 2000. Metabolic control of food intake and estrous cycles in syrian hamsters. I. Plasma insulin and leptin. Am. J. Physiol., 278: 476-485.

Sherwin, C.M. 1998. Voluntary wheel running: a review and novel interpretation. Anim. Behav., 56: 11-27.

Speakman, J.R., Gidney, A., Bett, J., Mitchell, I.P., Johnson, M.S. 2001. Limits to sustained energy intake IV: Effect of variation in food quality on lactating mice Mus musculus. J. Exp. Biol., 204: 1957-1965.

Sucajtys-Szulc, E., Goyke, E., Korczynska, J. 2008. Chronic food restriction differentially affects NPY mRNA level in neurons of the hypothalamus and in neurons that innervate liver. Neuroscience Letters, 433: 174-177

Terblanche, J.S., Janion, C., Chown, S.L. 2007. Variation in scorpion metabolic rate and rate-temperature relation: implications for the fundamental equation of the metabolic theory of ecology. J. Com. Pilation., 20: 1602-1612.

Tian, D.R., Li, X.D., Shi, Y.S. 2004. Changes of hypothalamic alpha-MSH and CART expression in diet-induced obse rats. Peptides, 25: 2147-2153.

Toshihiro, K., Akio, I.. 2001. Modest overexpression of neuropeptide Y in the brain leads to obesity after high-suerose feeding. Dlabetes, 50: 1206-1210.

Tracy, A.L., Clegg, D.J., Johnson, J.D., Davidson, T.L., Benoit, S.C. 2008. The melanocortin antagonist AgRP increases appetitive responding for a fat ,but not a carbohydrate, reinforcer. Pharmacol Biochem Behav, 89: 263-271.

Veloso, C., Bozinovic, F. 1993. Dietary and digestive constraints on basal energy metabolism in a small 

and/ or refed mice. Am. J. Physiol., 254: 11-16.

Woods, S.C., Seeley, R.J., Porte, J.D., Schwartz, M.W. 1998. Signals that regulate food intake and energy homeostasis. Science, 280:1378-1383.

Xiao, X.Q., Grove, K.L., Lau, S.Y., McWeeney, S., Smith, M.S. 2005. Deoxyribonucleic acid microarray analysis of gene expression pattern in the arcuate nucleus/ventromedial nucleus of hypothalamus during lactation. Endocrinology, 146: 4391-4398.

Yoo, S.B., Ryu, V., Park, E.Y. The arcuate NPY, POMC, and CART expressions responding to food deprivation are exaggerated in young female rats that experienced neonatal maternal separation. Neuropeptides, 2011, 45: 343-349.

Zhan, X.M., Li, Y.L., Wang, D.H. 2009. Effects of fasting and refeeding on body mass, thermogenesis and serum leptin in Brandt's voles (Lasiopodomys brandtii). Journal of Thermal Biology, 34: 237-243.

Zhang, L., Yang, F., Huang, C.M., Wang, Z.K., Zhu, W.L. 2015. The role of photoperiod on the expression of hypothalamic genes regulating appetite in Chevrier's field mouse (Apodemus chevrieri). Animal Biology, 65: 45-56.

Zhao, Z.J., Wang, D.H.. 2006. Effects of photoperiod on energy budgets and thermogenesis in Mongolian gerbils (Meriones unguiculatus). J. Therm. Biol., 31: 323-331

Zhao, Z.J., Wang, D.H. 2007. Effects of diet quality on energy budgets and thermogenesis in Brandt's voles. Comp. Biochem. Physiol., 148: 168-177. stochastic food deprivation and refeeding. Comp. Biochem. Physiol., 154: 84-91. 
Zhao, Z.J., Chi, Q.S., Cao, J., Wang, D.H. 2014. Seasonal changes of body mass and energy budget in Striped hamsters: the role of leptin. Physiological and Biochemical Zoology, 87: 245-256. Apodemus chevrieri in Hengduan mountains region during cold acclimation. Journal of Thermal Biology, 36: 81-186. digestive tract morphology in Apodemus chevrieri in Hengduan mountain region. Animal Biology, 62:463-478.

Zhu, W.L., Mu, Y., Zhang, H., Zhang, L., Wang, Z.K. 2013. Effects of food restriction on body mass,

Zhu, W.L., Mu, Y., Zhang, H., Gao, W.R., Zhang, L., Wang, Z.K. 2014. Effects of random food deprivation on body mass, behavior and serum leptin levels in Eothenomys miletus (Mammalia: Rodentia: Cricetidea). Italian Journal of Zoology, 81: 227-234. 
2 Fig 1 A time line showing which days food deprivation occurred and which day each measurement was

3 taken in Apodemus chevrieri. An asterisk put below every food deprived day." R", refeeding.

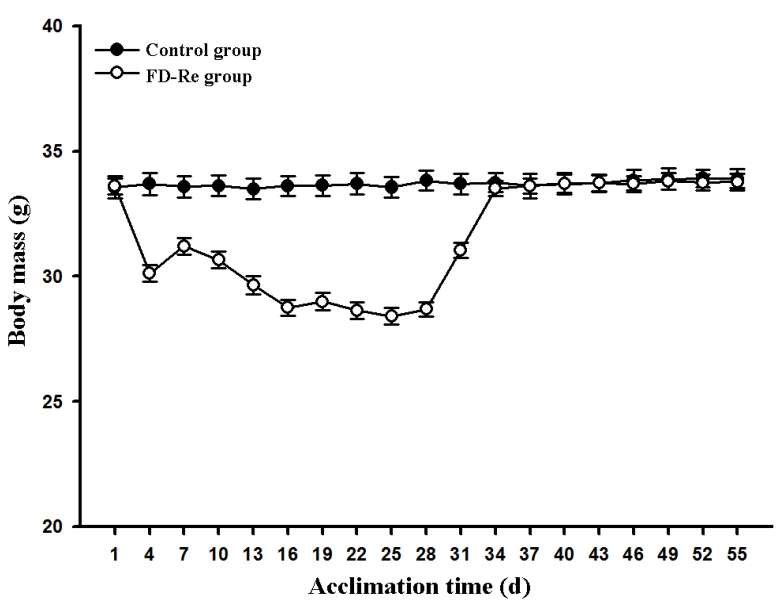

Fig 2 Changes in body mass of Apodemus chevrieri in response to random food deprivation and ad libitum re-feeding.

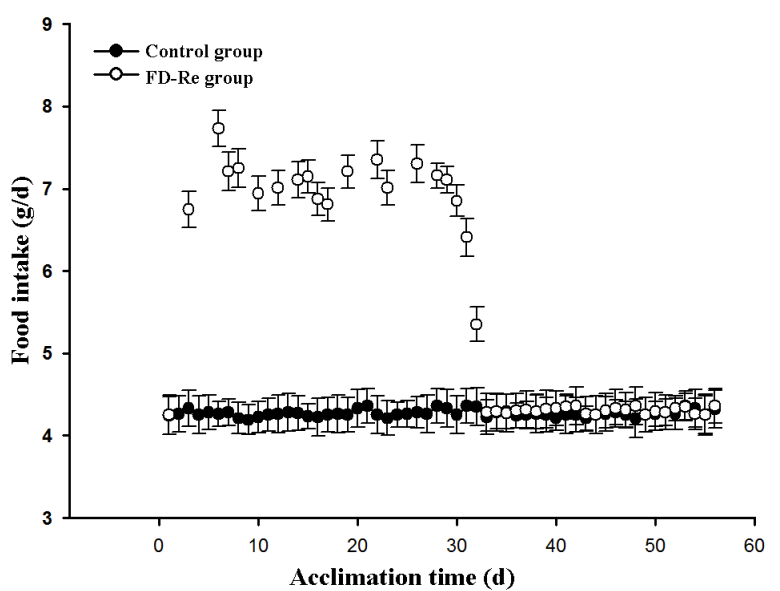

Fig 3 Changes in food intake of Apodemus chevrieri in response to random food deprivation and ad libitum re-feeding. 


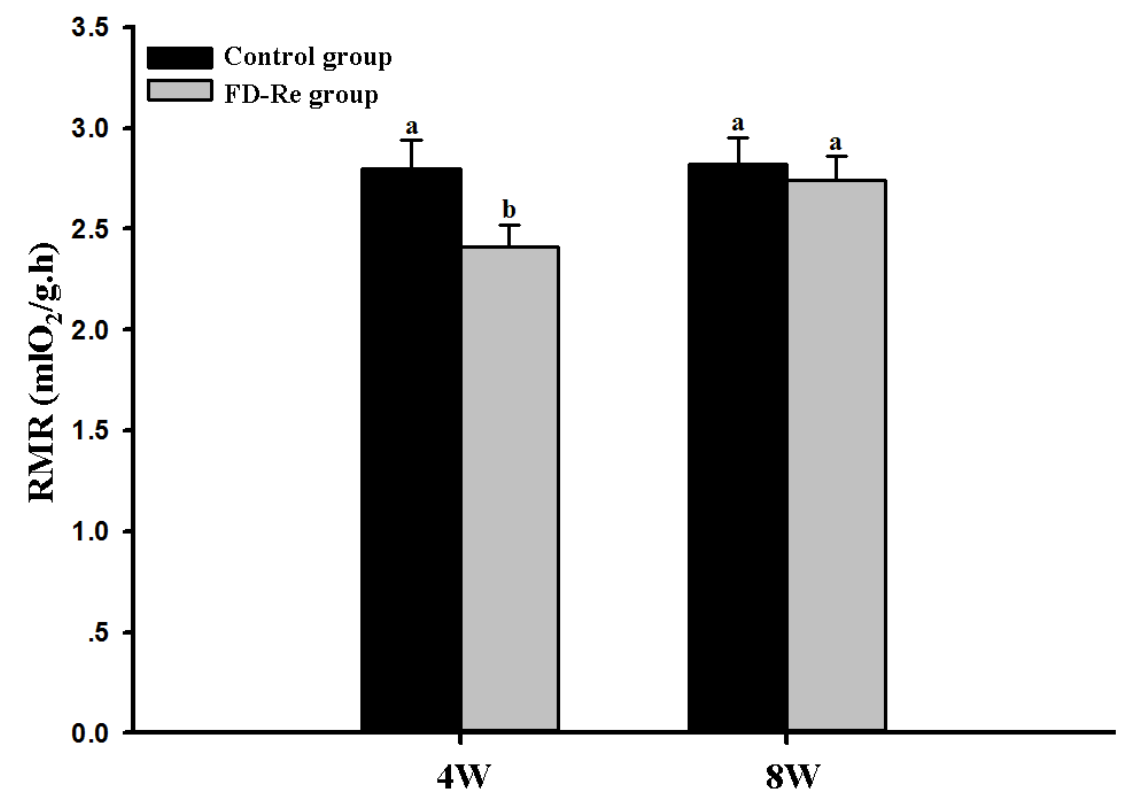

Fig 4 Changes in RMR of Apodemus chevrieri in response to random food deprivation and ad libitum re-feeding. Different superscripts in each row indicate significant difference $(P<0.05)$.

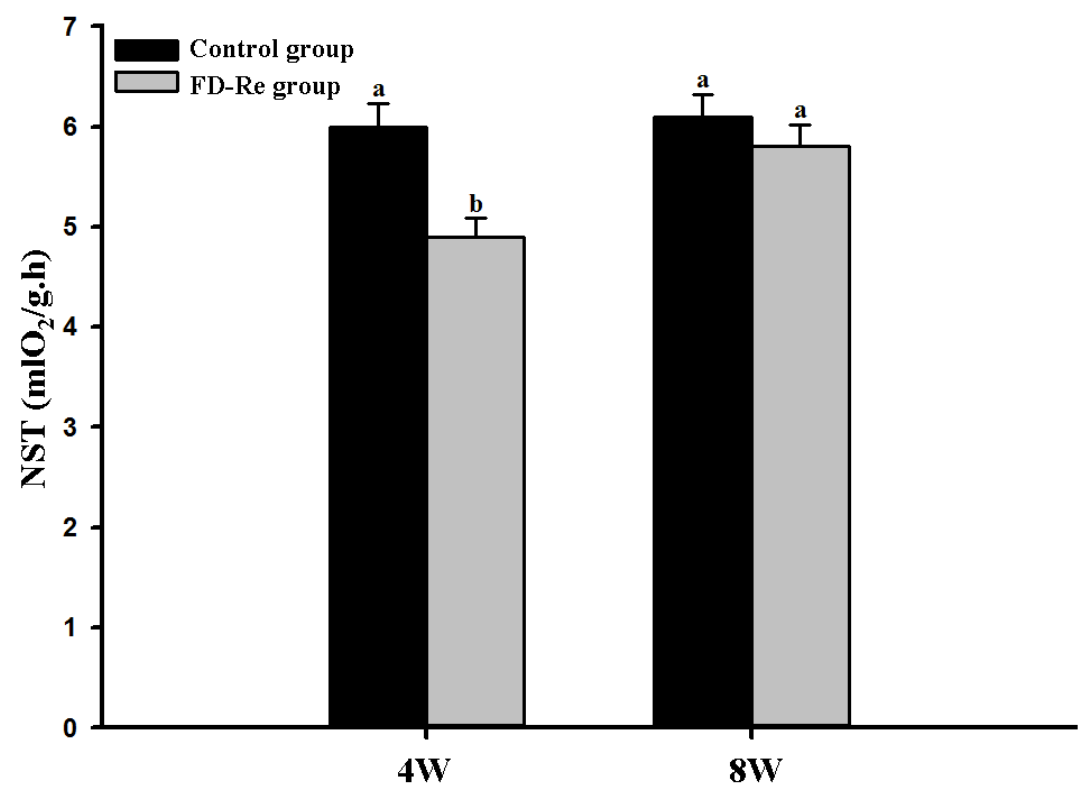

Fig 5 Changes in NST of Apodemus chevrieri in response to random food deprivation and ad libitum

re-feeding. Different superscripts in each row indicate significant difference $(P<0.05)$. 

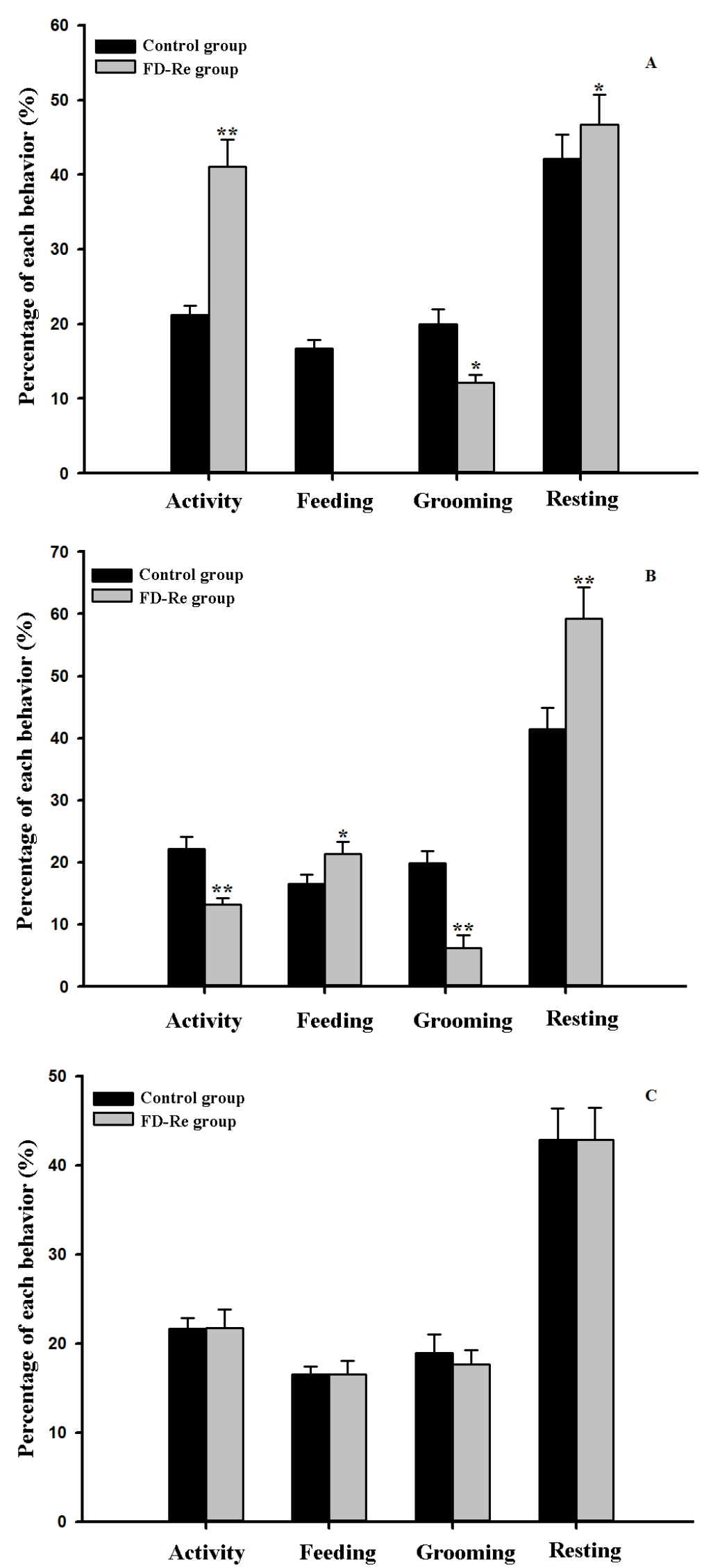

2 Fig 6 Changes in general activity, feeding, grooming and resting behavior of Apodemus chevrieri on food

deprived day (A), on ad libitum day (B) and on re-feeding day (C). $* \mathrm{P}<0.05, * * \mathrm{P}<0.01$ 


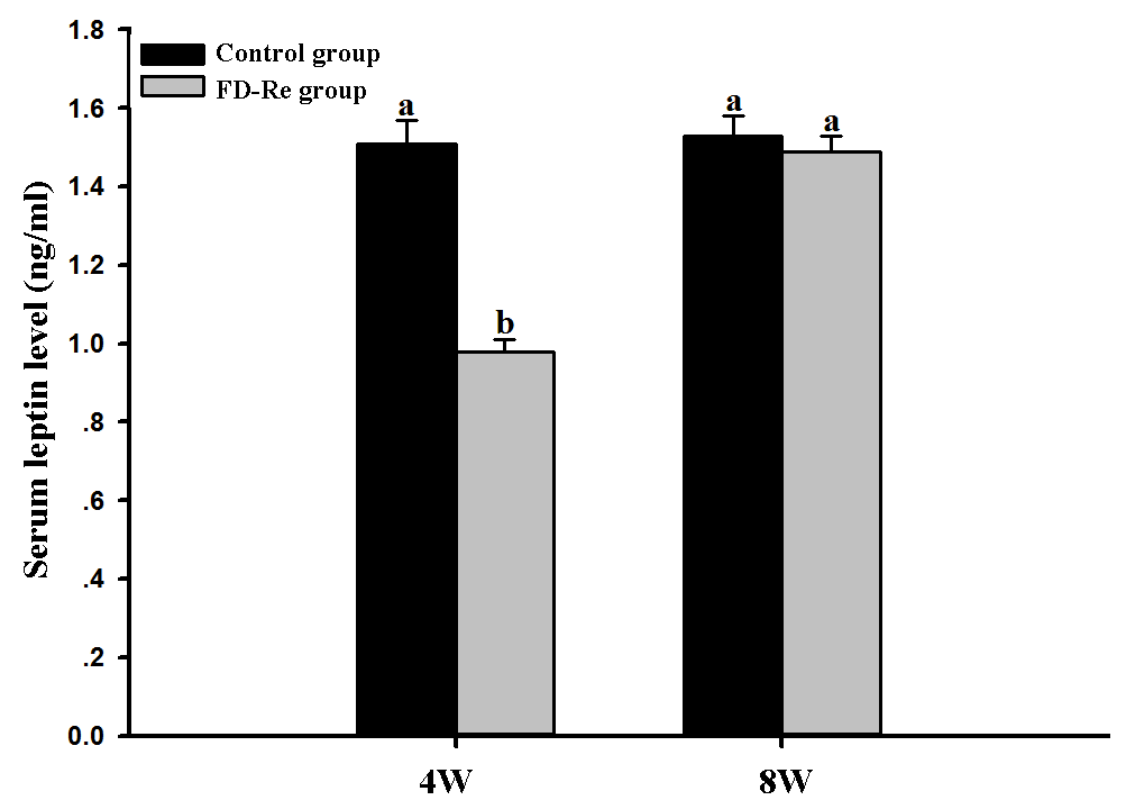

2 Fig 7 Changes in serum leptin levels of Apodemus chevrieri in response to random food deprivation and ad libitum re-feeding. Different superscripts in each row indicate significant difference $(P<0.05)$.

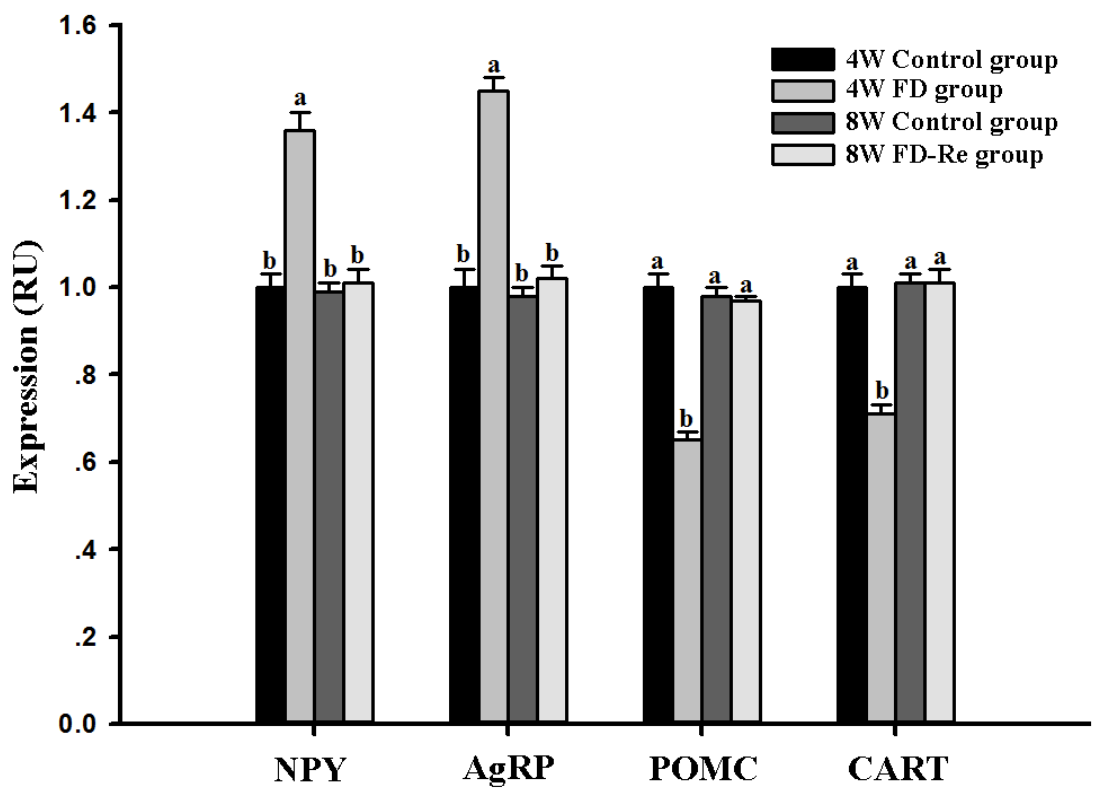

Fig 8 Changes in NPY, AgRP, POMC and CART expression of Apodemus chevrieri in response to random food deprivation and ad libitum re-feeding. Different superscripts in each row indicate significant difference $(\mathbf{P}<\mathbf{0 . 0 5})$.

Table 2 Effects of food deprivation and ad libitum re-feeding on body composition in Apodemus chevrieri.

\begin{tabular}{ccccc}
\hline Parameters & 4W Control & 4W FD group & 8W Control & 8W FD-Re group \\
\hline Body mass $(g)$ & $33.72 \pm 0.22^{\mathrm{a}}$ & $28.32 \pm 0.19^{\mathrm{b}}$ & $33.75 \pm 0.25^{\mathrm{a}}$ & $33.77 \pm 0.21^{\mathrm{a}}$ \\
Wet Carcass mass $(\mathrm{g})$ & $23.61 \pm 0.22^{\mathrm{a}}$ & $20.36 \pm 0.17^{\mathrm{b}}$ & $24.51 \pm 0.15^{\mathrm{a}}$ & $23.45 \pm 0.18^{\mathrm{a}}$ \\
Dry carcass mass $(\mathrm{g})$ & $7.89 \pm 0.21^{\mathrm{a}}$ & $6.84 \pm 0.12^{\mathrm{b}}$ & $8.01 \pm 0.19^{\mathrm{a}}$ & $7.91 \pm 0.17^{\mathrm{a}}$ \\
Body fat mass $(\mathrm{g})$ & $4.56 \pm 0.12^{\mathrm{a}}$ & $2.42 \pm 0.09^{\mathrm{b}}$ & $4.71 \pm 0.13^{\mathrm{a}}$ & $4.51 \pm 0.10^{\mathrm{a}}$ \\
Heart & & & \\
\hline
\end{tabular}




\begin{tabular}{ccccc}
\hline Wet mass $(g)$ & $0.256 \pm 0.013^{\mathrm{a}}$ & $0.238 \pm 0.012^{\mathrm{b}}$ & $0.249 \pm 0.008^{\mathrm{a}}$ & $0.234 \pm 0.009^{\mathrm{a}}$ \\
Dry mass(g) & $0.061 \pm 0.003$ & $0.058 \pm 0.006$ & $0.063 \pm 0.002$ & $0.060 \pm 0.003$ \\
Lungs & & & & \\
Wet mass(g) & $0.224 \pm 0.016$ & $0.218 \pm 0.012$ & $0.228 \pm 0.011$ & $0.221 \pm 0.009$ \\
Dry mass(g) & $0.064 \pm 0.004$ & $0.061 \pm 0.002$ & $0.059 \pm 0.003$ & $0.058 \pm 0.004$ \\
Liver & & & & \\
Wet mass(g) & $2.231 \pm 0.142$ & $1.672 \pm 0.126$ & $2.241 \pm 0.153$ & $2.204 \pm 0.114$ \\
Dry mass(g) & $0.876 \pm 0.024$ & $0.842 \pm 0.015$ & $0.861 \pm 0.013$ & $0.859 \pm 0.011$ \\
Brown adipose tissue & & & & \\
Wet mass(g) & $0.214 \pm 0.013^{\mathrm{a}}$ & $0.152 \pm 0.009^{\mathrm{b}}$ & $0.211 \pm 0.012^{\mathrm{a}}$ & $0.198 \pm 0.011^{\mathrm{a}}$ \\
Dry mass(g) & $0.019 \pm 0.001$ & $0.016 \pm 0.001$ & $0.020 \pm 0.001$ & $0.018 \pm 0.001$ \\
Kidney & & & & \\
Wet mass(g) & $0.452 \pm 0.026$ & $0.421 \pm 0.021$ & $0.461 \pm 0.019$ & $0.435 \pm 0.016$ \\
Dry mass(g) & $0.136 \pm 0.005$ & $0.122 \pm 0.006$ & $0.139 \pm 0.008$ & $0.129 \pm 0.008$ \\
Spleen & & & & \\
Wet mass(g) & $0.059 \pm 0.002$ & $0.061 \pm 0.003$ & $0.064 \pm 0.003$ & $0.058 \pm 0.002$ \\
Dry mass(g) & $0.015 \pm 0.001$ & $0.013 \pm 0.001$ & $0.016 \pm 0.001$ & $0.015 \pm 0.001$ \\
\hline
\end{tabular}

1 Different superscripts in each row indicate significant difference $(\mathbf{P}<0.05)$.

2

3 Table 3 Effects of food deprivation and ad libitum re-feeding on gastrointestinal tract in Apodemus chevrieri.

\begin{tabular}{|c|c|c|c|c|}
\hline Parameters & 4W Control & 4W FD group & 8W Control & 8W FD-Re group \\
\hline \multicolumn{5}{|l|}{ Stomach } \\
\hline Length(mm) & $22.71 \pm 0.126$ & $23.60 \pm 0.152$ & $22.61 \pm 0.162$ & $22.19 \pm 0.146$ \\
\hline Mass with content $(\mathrm{g})$ & $0.613 \pm 0.162^{b}$ & $0.861 \pm 0.135^{\mathrm{a}}$ & $0.621 \pm 0.122^{b}$ & $0.638 \pm 0.146^{b}$ \\
\hline Wet mass $(g)$ & $0.251 \pm 0.019$ & $0.259 \pm 0.018$ & $0.272 \pm 0.014$ & $0.260 \pm 0.012$ \\
\hline Dry mass(g) & $0.064 \pm 0.003$ & $0.065 \pm 0.002$ & $0.063 \pm 0.002$ & $0.061 \pm 0.004$ \\
\hline \multicolumn{5}{|l|}{ Small intestine } \\
\hline Length(mm) & $356.21 \pm 23.21^{\mathrm{b}}$ & $370.16 \pm 19.87^{\mathrm{a}}$ & $361.25 \pm 21.26^{b}$ & $365.23 \pm 23.69^{b}$ \\
\hline Mass with content(g) & $1.123 \pm 0.102^{b}$ & $1.452 \pm 0.095^{\mathrm{a}}$ & $1.095 \pm 0.087^{\mathrm{b}}$ & $1.114 \pm 0.065^{b}$ \\
\hline Wet mass $(\mathrm{g})$ & $0.451 \pm 0.016$ & $0.463 \pm 0.011$ & $0.459 \pm 0.021$ & $0.456 \pm 0.019$ \\
\hline Dry mass(g) & $0.089 \pm 0.002$ & $0.091 \pm 0.002$ & $0.085 \pm 0.003$ & $0.088 \pm 0.002$ \\
\hline \multicolumn{5}{|l|}{ Large intestine } \\
\hline Length(mm) & $184.23 \pm 12.36$ & $192.32 \pm 18.32$ & $186.65 \pm 13.02$ & $187.54 \pm 14.65$ \\
\hline Mass with content(g) & $0.325 \pm 0.019^{b}$ & $0.474 \pm 0.026^{\mathrm{a}}$ & $0.326 \pm 0.022^{b}$ & $0.331 \pm 0.025^{b}$ \\
\hline Wet mass $(g)$ & $0.221 \pm 0.011$ & $0.236 \pm 0.016$ & $0.219 \pm 0.0 .009$ & $0.223 \pm 0.012$ \\
\hline Dry mass(g) & $0.049 \pm 0.003$ & $0.052 \pm 0.002$ & $0.048 \pm 0.004$ & $0.051 \pm 0.003$ \\
\hline \multicolumn{5}{|l|}{ Cecum } \\
\hline Length(mm) & $96.32 \pm 6.54$ & $98.25 \pm 5.62$ & $93.98 \pm 6.58$ & $94.63 \pm 6.31$ \\
\hline Mass with content(g) & $0.865 \pm 0.033^{b}$ & $0.984 \pm 0.035^{\mathrm{a}}$ & $0.859 \pm 0.024^{\mathrm{b}}$ & $0.864 \pm 0.021^{b}$ \\
\hline Wet mass $(\mathrm{g})$ & $0.286 \pm 0.016$ & $0.291 \pm 0.025$ & $0.276 \pm 0.021$ & $0.284 \pm 0.013$ \\
\hline Dry mass(g) & $0.051 \pm 0.002$ & $0.053 \pm 0.003$ & $0.049 \pm 0.004$ & $0.051 \pm 0.004$ \\
\hline
\end{tabular}

4 Different superscripts in each row indicate significant difference $(\mathrm{P}<0.05)$. 